Health Informatics - An International Journal (HIIJ) Vol.4, No.3/4, November 2015

\title{
THE 4 R'S - REASON, REDCAP, REVIEW AND RESEARCH - IN A LARGE HEALTHCARE ORGANIZATION
}

\author{
Christopher Bain ${ }^{1,2}$, Annie Gilbert ${ }^{3}$, Bismi Jomon ${ }^{3}$, Robin Thompson ${ }^{3}$, David \\ Kelly $^{3}$ and Chris Mac Manus ${ }^{4}$ \\ ${ }^{1}$ Faculty of Information Technology, Monash University. Clayton, Vic. Australia \\ ${ }^{2}$ Health Information Services, Mercy Hospital for Women. Heidelberg, Vic. Australia \\ ${ }^{3}$ Applications and Knowledge Management Department, Alfred Health. \\ Melbourne. Vic. Australia \\ ${ }^{4}$ Ozescribe. Glen Iris, Vic, Australia
}

\begin{abstract}
This paper outlines the journey of a large Australian academic health service in relation to the acquisition, installation and roll out of the REDCap platform (RCP) for the betterment of clinical review (clinical audit) and research data collection. The main aims of the acquisition of the platform were to facilitate data collection and management for audit and research across the organization in a more sustainable way than had previously been possible. We found the platform to be easily installed and maintained. There was rapid uptake of the platform by a range of health service stakeholders across the audit, research and operational domains. We were also able to successfully integrate data from our corporate clinical data environment, The REASON Discovery Platform ${ }^{R}$ (REASON) into selected REDCap "applications" using the Dynamic Data Pull (DDP) functionality it provides. In summary the acquisition and installation of REDCap at our health service has been hugely successful and has provided a great facility for use by a large number of organizational stakeholders going forwards into the future.
\end{abstract}

\section{KEYWORDS}

Hospital, REDCap, REASON, audit, research

\section{INTRODUCTION}

There are a number of challenges facing large healthcare organizations, particularly those where research and audit are key agenda items for the organization.

One such challenge is how to balance the operational needs of functional systems and data collections, often tailored to highly specialized health service delivery areas (eg - cardiology versus obstetrics), with a corporate need to save money and provide good information technology (IT) governance, often through standardisation and consolidation. This can lead to tensions between somewhat autonomous business areas and the centralised corporate functions of information management (IM) and IT. In this case study we will examine how our organization set about managing this tension - particularly, but not only, in relation to clinical audit and research needs.

Our health service, Alfred Health (AH) [1], has 3 main hospital campuses and several smaller satellite facilities (including psychiatric outreach clinics and a specialised sexual health centre) under its control, as well as many ambulatory services. It also provides state-wide referral services in the areas of adult organ transplantation, adult burns and adult trauma.

DOI: $10.5121 /$ hiij.2015.4402 
Health Informatics - An International Journal (HIIJ) Vol.4, No.3/4, November 2015

The original setting for this work is the creation of a Health Informatics (HI) department at the health service in early 2011 . The department was charged with assuming responsibility for the technical development and management of the corporate data and reporting infrastructure, whilst another separate key business unit was charged with the responsibility for delivering data and reporting off the infrastructure. In the mid part of 2011, HI along with Health Information Services (HIS), Information Technology Services (ITS) and the Australian Centre for Health Innovation was brought under a single new business division - the Information Development Division (IDD). It was the vision of the new divisional head to continue to develop this technical infrastructure as part of a broader plan. After several corporate restructures, this work is now being continued in the recently created Applications and Knowledge Management (AKM) Department.

\section{BACKGROUND}

Often the "big data" paradigm is viewed as a very modern phenomenon, in part driven by recent changes in technology that support it, and in part by the vast array of machine generated data (eg from medical devices) now available to us for leveraging. It could be argued however that healthcare, with its large numbers of patients and decades of detailed history about them in variety of formats (in many cases), has been operating in such a paradigm for many years. How well it has done in efficiently handling such large volumes of data is a separate question however. Although working with, and making sense of, "big data" in the more modern sense of the term, is not without its dangers [2], the benefits of its use are thought to be significant [3]. Some of the purported advantages of the "big data" paradigm are the ability to mine data sets for patterns, identify uncommon events and to unearth interesting or valuable insights

As previously described in the literature [4], we have constructed a platform in this paradigm called The REASON Discovery Platform ${ }^{\circledR}$. We have also previously described some of the actual and potential value from the platform [5-9], including the development of a web based cohort identification tool [10], a prototype image analysis platform [11] and automated data collation and transmission to clinical registries [12-14]

It can be difficult for readers to get a full appreciation of what is being attempted through the construction of the REASON platform as the means to address this technical need. There are 2 United States (US) based examples outlined below, that allow the reader to get a sense of the amount of work done to date to establish this infrastructure, and the direction of travel of the platform.

One US initiative is "Informatics for Integrating Biology and the Bedside" (i2b2), although this operates under a different kind of governance model to our platform, and arguably has a broader reach [15]. One of the primary aims of the $\mathrm{i} 2 \mathrm{~b} 2$ Centre is in "developing a scalable computational framework to address the bottleneck limiting the translation of genomic findings and hypotheses in model systems relevant to human health". This initiative is well known internationally and there are even competitions to analyse data provided from the platform.

The REASON platform however, is most closely aligned to the Stanford - based STRIDE (Stanford Translational Research Integrated Database Environment). STRIDE "is a research and development project at Stanford University to create a standards-based informatics platform supporting clinical and translational research." [16]. There has been evidence published in the international literature pertaining to the benefits to health care processes, and patients, of such a platform [17]. In order to augment the scope and benefits of REASON, as well as to deal with the 
issue of excessive application diversity, a corporate decision was made to acquire the REDCap Platform (RCP) [18-19].

The RCP (Figure 1) is an open-source, secure web application that can be used for data capture and analysis in different ways. This application is developed by Vanderbilt University and is available to institutional partners at no cost. AH has been a member of the REDCAP consortium based at Vanderbilt since early 2014. With minimal effort and training, any user can access this application to build and manage online data collection instruments and analyse them at their own pace. The potential advantage of using this application is that the data is stored centrally and access is secured and controlled by active directory login [20]. The real benefit to organisations around the use of the RCP is that provides a pathway to reduce the use of standalone legacy applications like Microsoft Access 97 databases. In addition it is easy to access and use with a range of different end-point devices like iPads, Motion tablets and desktop PC's.

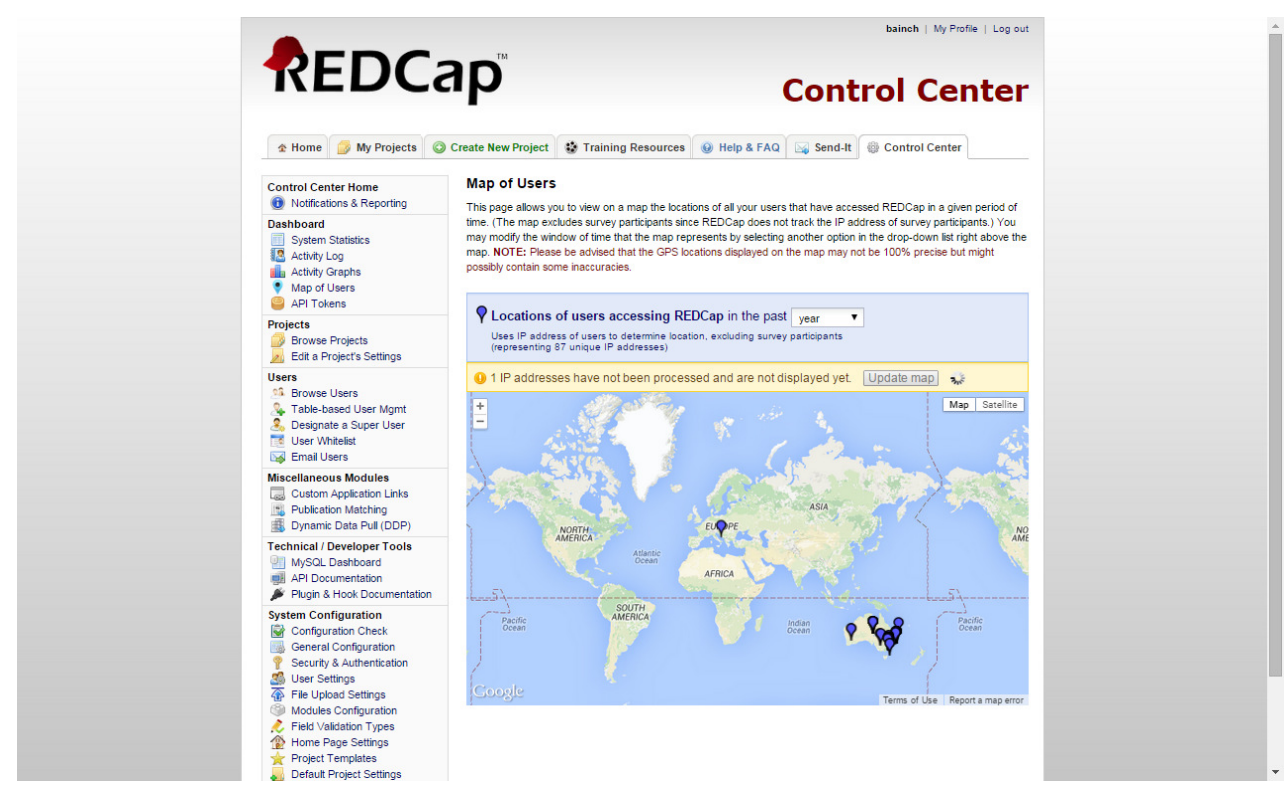

Figure 1. Map based view of IP addresses of users accessing AH REDCap Platform (RCP)

In this article, through a case study approach, we will examine how our organisation has dealt with the big data deluge, and the issue of excessive numbers of software applications and standalone databases, through the use of the RCP.

\section{METHOD}

\subsection{Pre-Acquisition of the RCP}

At AH there are 7000 or more employees, 3 main hospitals and multiple satellite sites, several attached internationally known research facilities and a close affiliation with several universities most notably Monash University. In addition the health service contributes to about 90 clinical registries. As a result there are a multitude of needs when it comes to data collection, management and analysis

As a direct result of this, and also driven by suboptimal information and technology governance in the past, multiple point information solutions have developed over time, often run 
independently by individual departments. This has had a number of adverse effects - for example there are now many thousands of standalone Microsoft Access databases across the organization which, whilst filling a need and providing benefits to the immediate users, also introduce risks to the organization such as incompatibilities between old software environments - eg MS Access 97 (officially out of support 11 years ago) [21] and newer operating systems such as Windows 7. A scan of the 4 key organizational file servers at the time of writing revealed 13,925 MS Access databases on these servers alone, $92 \%$ of which were actually older MS Access versions (Access 97, XP or 2002). This scan does not include the MS Access databases solely located on hardware end points like PC's. In some cases these standalone systems gave matured to be proxy electronic medical records (EMRs), and in one case such a system needed to be urgently decommissioned and replaced due to a key man risk being realised.

One of the principles used by the IDD in order to try to meet these needs was to aim to use platform level solutions where possible, to address multiple stakeholder needs in a cost effective, extensible and sustainable way. It is in this context, and in the setting of REASON having been established, that when the relevant leaders became aware of the existence of REDCap, and it use in a large research facility across town, that investigations about the acquisition of the RCP for AH commenced

\subsection{RCP Acquisition and Pilot}

We became aware that the Murdoch Children's Research Institute (MCRI) [22] had used REDCap for several years. We did a virtual introduction with the key contact there (using the contact details kept by the REDCap consortium) in order to explore this. We then met the REDCap administrator at the MCRI and were immediately impressed both by what we saw, and how the MCRI were using and running REDCap. We felt that many elements of their model would be fairly easy to establish at $\mathrm{AH}$, and that the platform was eminently suited to addressing some core data and information needs at $\mathrm{AH}$.

A technical investigation was then performed in conjunction with the other IDD Departments to assess the technical requirements needed to host the platform, and the ease of acquiring suitable hardware. One this was done, the Executive Director of the IDD approved the acquisition of REDCap and signed off on the REDCap consortium agreement (March 2014) so that a pilot of the platform could commence.

A pilot was run in the organisation from mid-2014. This pilot focused on 3 key research and audit projects - the Patient Experience Survey (PES), the Limiting IV Chloride to Reduce Acute Kidney Injury (LICRA) clinical trial [23] and the Severe Asthma Clinic Survey. In the section (Section 4) that follows we will focus on the results of some specific pieces of work using REDCap in the setting of the REASON platform and our corporate strategies in this area.

\subsection{Operationalization and Advanced Usage of the RCP}

There were 3 key evaluation principles underpinning the pilot of the RCP at $\mathrm{AH}$. These were:

- could the platform be successful installed and connected to other hospital systems (eg Active Directory [20] for authentication)

- could users be trained to successfully create REDCap applications and collect data in them and

- could the users then get access to that data for their desired purpose. 
It was fairly clear after 6 months of usage of the platform that all of these criteria had been easily fulfilled. As a result, a formal decision was made to operationalise the system and make it part of the technology landscape at $\mathrm{AH}$. In turn, several key undertakings were performed to facilitate this operationalisation

- responsibility for the platform was established in the AKM department

- the Dynamic Data Pull (DDP) [18] functionality of REDCap was instantiated

- the routine nightly upload of all REDCap Database (DB) data into REASON was established

- access requests for REDCap were set us as a usable service in the IT service catalogue, and

- more powerful and robust hardware was sought to support the expected growth in usage of the RCP.

In addition, towards the middle of 2015, a formal disaster recovery approach for REDCap was designed and implemented, over and above the routine REDCap DB backups.

\section{RESULTS}

In this section of the paper we will examine the outcome of the RCP pilot, and some of the achievements with the system since it was subsequently formally operationalized at $\mathrm{AH}$.

\subsection{The Patient Experience Survey}

As described above, the Patient Experience Survey (PES) was one of the original projects created on the AH RCP as part of the pilot.

PES (Figure 2) is about capturing patient experience across the health service. The study captured all areas of the patient experience including some descriptive features of the patients themselves, the area of the hospital they were in, the service they attended or utilised, and their feedback about the quality of the service or care that they received. There are now more than 3,500 patient responses, from the three different campuses, which were collected by volunteers in the PES REDCap "application".

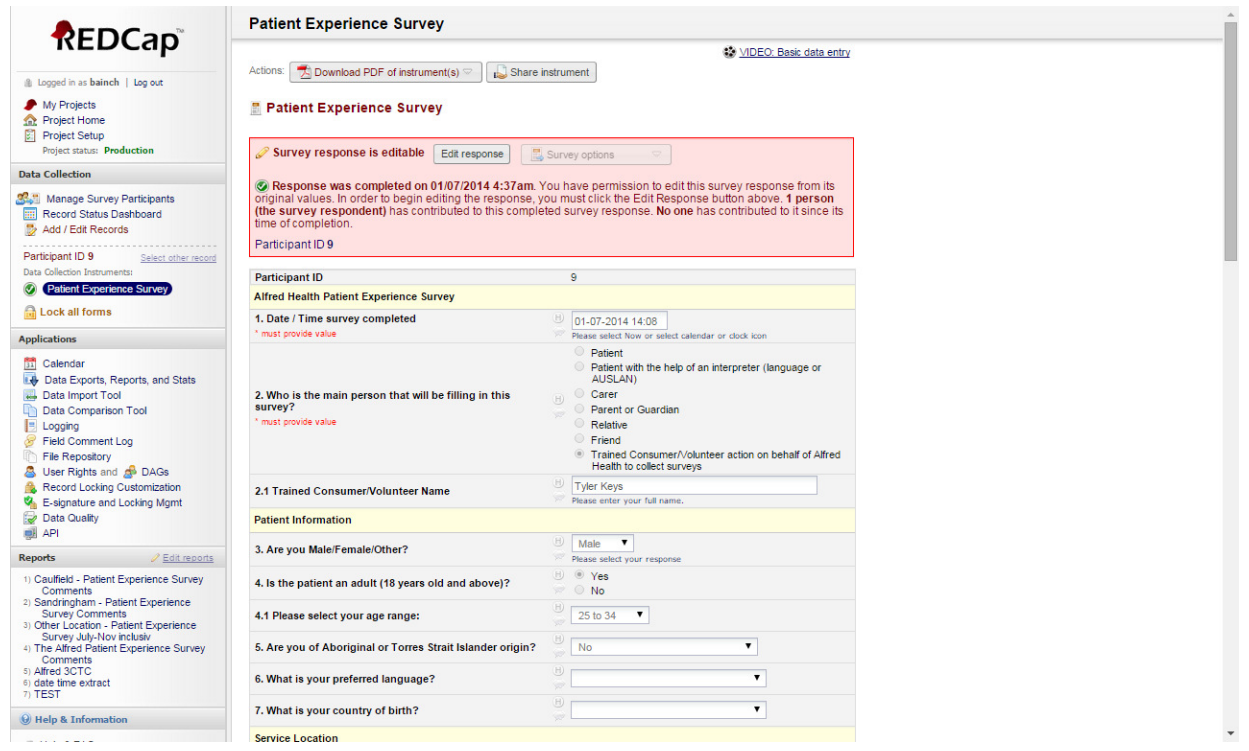

Figure 2. Patient Experience Survey (PES) in the AH RCP Instance 
With the recent progress in technology and infrastructure at $\mathrm{AH}$, it ought soon be possible that each and every area of the organisation will have wireless connectivity, and hence feedback could be collected from patients or carers any physical location across the various health service campuses.

\subsection{The LICRA Study}

The LICRA study is a research study being run by the Anaesthesia Department at AH [22]. The study aims to test the impact of a strategy of peri-operative chloride-restriction through IV fluid therapy on incidence of acute kidney injury after cardiothoracic surgery. There was an existing paper based case report form (CRF) that was replaced by a development in REDCap by the anaesthetics research team as part of a pilot study for LICRA. There were 13 data collection instruments and a total of 560 fields as part of this LICRA pilot study. It took approximately 1 hour per record to complete the paper based data collection instruments.

The AKM Department subsequently conducted an analysis of the fields to be collected for the LICRA study and ascertained that many of these were already collected or generated in the Cerner Millennium environment [24], AH's EMR System. As such, these fields were extracted and available from the REASON Discovery Platform which receives much of the Millennium data routinely on a daily basis. An extract was written to retrieve these files from REASON, and combine it with the additional data entered through REDCap, to provide a consolidated dataset for the researcher. After extensive testing, the number of data collection instruments in REDCap was reduced to 8 with only 249 fields. Part of the validation process highlighted some manual error occurred in the original data collection approach, either due to transcription problems, or updates to the EMR post CRF completion (Figure 3). Providing a combined electronic dataset in this way resulted in a 50\% reduction in data entry time, and an improvement in data quality. The final dataset is being analysed in separate statistics package.

All projects that have data capture requirements for registry submissions at $\mathrm{AH}$, and have data collection gaps identified or need the facilitation of data collection supported, could consider REDCap to support this process using a similar approach to what we have outlined here, so only data that is not already in existence electronically is then collected - rather than re-collecting or re-recording data already present in other hospital systems.

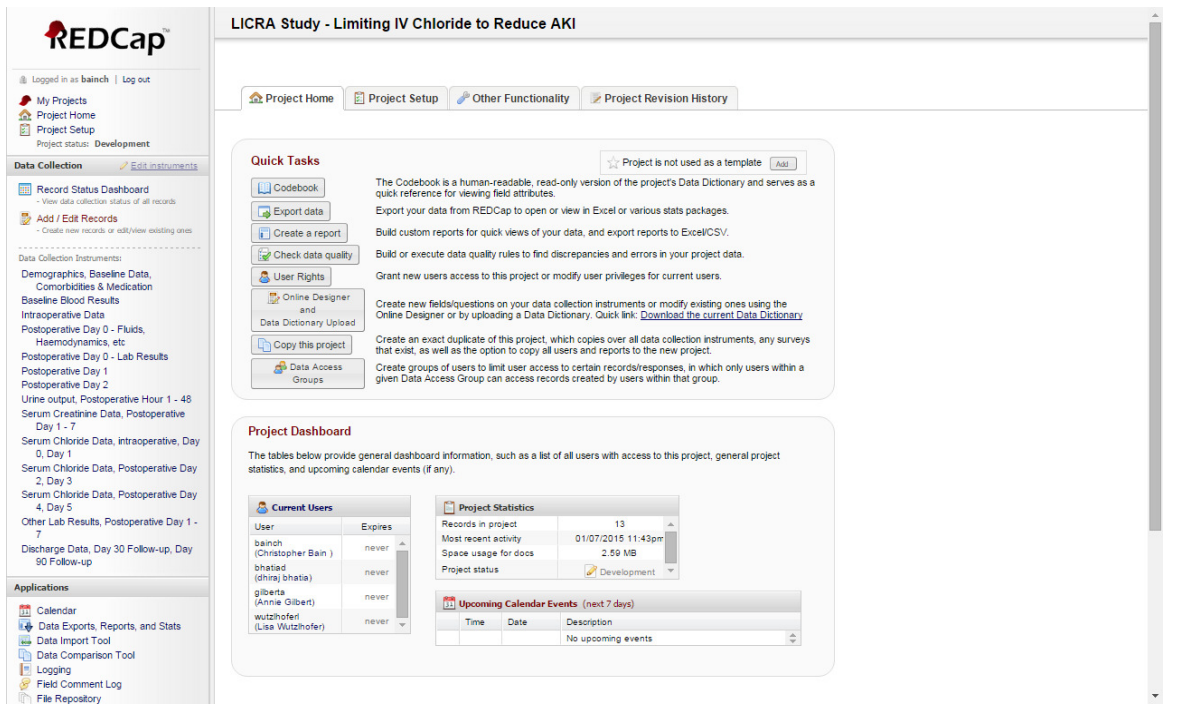

Figure 3. LICRA project in the AH RCP Instance 


\subsection{Key Technical Achievements}

Several projects are currently using the Dynamic Data Pull (DDP) functionality of REDCap to validate the patient details - in the Team and Patient Assessment System (TAPAS) project in General Medicine for example, plus it will also be used in some upcoming nursing audit projects, including the re-creation of the Point of Care (POC) audit [7-8] in REDCap. The DDP feature allows data to be imported into the data collection instrument from an external source. It operates by communicating between web services via HTTP/HTTPS. In our case, the patient first name, last name, gender and date of birth are retrieved when the patient's medical record number is entered into the relevant REDCap collection instrument.

\subsection{Overall REDCap Usage at AH}

As can be seen in Figure 4 and Tables 1 and 2, the RCP provides very a comprehensive suite of information pertaining to the use of the local RCP instance. This information has been very valuable in managing the platform and in getting a clear picture of the uptake of the RCP across the health service.

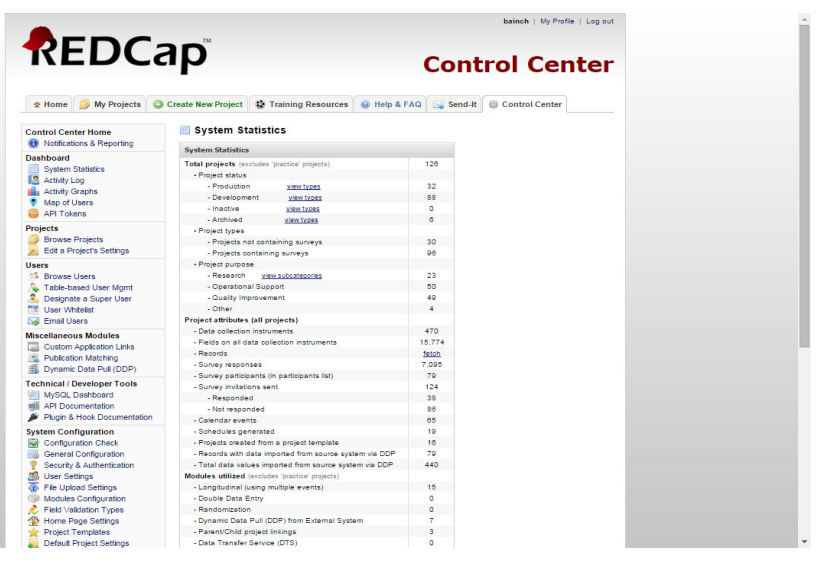

Figure 4. AH RCP Instance Statistics

At the time of writing there were about 120 projects ("applications") on the RCP and 130 users with access to the platform, and all were active -as opposed to inactive or suspended users. Users are people with security accounts that allow them then to access the platform and potentially develop REDCap projects, and this number does not include end-users who may complete surveys, for themselves or on behalf of others, that are housed on the platform.

When viewed from an "event log" perspective (Table 1) there had been over 35,000 events recorded by the system in the last month as at the time of writing.

Table 1. Event based usage of the AH RCP Instance.

\begin{tabular}{|l|l|}
\hline Parameter & Value \\
\hline Total logged events & 138,045 \\
\hline - Past 30 minutes & 22 \\
\hline - Today & 1,418 \\
\hline - Past 7 days & 8,208 \\
\hline - Past 30 days & 35,505 \\
\hline
\end{tabular}


There were also more than 15,700 data fields being used across 470 data instruments in the $\mathrm{AH}$ instance of the RCP (Table 2) at the time of writing, as well as over 13,000 records of different kinds across the 120 plus projects.

Table 2. Record and content based usage of the AH RCP Instance.

\begin{tabular}{|l|l|}
\hline Parameter & Value \\
\hline Data collection instruments & 470 \\
\hline Fields on all data collection instruments & 15,774 \\
\hline Records & 13,035 \\
\hline Survey responses & 7,095 \\
\hline Survey participants (in participants list) & 79 \\
\hline Survey invitations sent & 124 \\
\hline$\quad$ - Responded & 38 \\
\hline$\quad$ - Not responded & 86 \\
\hline Calendar events & 65 \\
\hline Schedules generated & 19 \\
\hline Projects created from a project template & 16 \\
\hline Records with data imported from source system via DDP & 79 \\
\hline Total data values imported from source system via DDP & 440 \\
\hline
\end{tabular}

\section{DISCUSSION}

\subsection{An Overview}

The pilot of the RCP at AH was successfully completed in December 2014. The uptake of REDCap in the organisation has been predominantly via word of mouth, rather than active advertising. In all cases there has been positive interest and engagement with the projects developed to date on the RCP - 75\% of which are surveys and $25 \%$ are data collection instruments In terms of the purposes of the projects, $40 \%$ are for operational support, $40 \%$ for quality improvement and $18 \%$ are for research. The potential advantage of using this application is that the data is stored centrally and access is secured and controlled by active directory logins. The data collected through this tool is also uploaded on a nightly basis to the REASON platform for fast and flexible reporting and research purposes.

The work has been so successful that now the AH Ethics Committee (EC) has also approved the RCP as an appropriate organisational wide data collection method wherever suitable. The AKM Business Services team then govern the process to create and release projects into production, and they also train the relevant super users. In addition we have implemented a support model where this team review the applications for REDCap use, ensure EC approval is obtained where required (eg- for project requests not coming through the AH EC), provide locations for the online training and assist in design, and implementation, of REDCap applications where required. We have found that with minimal effort and training, any user can use this application to build and manage online data collection instruments and analyse the data from them (at a high level) at their own pace. The user is not subject to other IT processes or beholden to other IT priorities. The challenge with this is where the users are too busy to create their own survey, although contractor REDCap developer services are available to them if they can find funding. Enabling users to be able to develop their own surveys and data collection instruments has been a very positive process. Non-technical users are able to develop and utilise the online designer, branching logic and calculated fields easily. 
One of the real benefits to our organisation in using the RCP is a reduction in the number of standalone legacy applications like MS Access databases, and its ease of use with a range of different types of devices like iPads and Motion tablets. It is now much easier to acquire and leverage "big data" whilst simultaneously preventing further growth in the numbers of standalone applications around the business. In addition, because of the RCP, there is now also a standardised approach supported for capturing quality of care data separately to the EMR, ensuring that the EMR's focus is the actual clinical care of patients and the workflow and decision support around that care. Finally, findings from research conducted in REDCap can be used as an impetus to further research, and cross collaboration between research teams can be easily facilitated.

AKM are also hoping to expand our use of REDCap to include more advanced functionality including API's and utilising the Plugin and Hook functions. Technical training about some of these RCP capabilities will be addressed at the US REDCap conference being held in late 2015.

\subsection{Lessons Learned}

There have been cultural challenges within the organisation to be addressed through this process, where there is the perception of data, knowledge and control are lost if a central standardised methodology is used for data collection. This is being addressed by individual meetings and discussion, and future REDCap seminars to promote success stories where REDCap has been utilised. It is also critical that REDCap is not perceived as a replacement to data capture that is required in the EMR. A policy is being developed that patient data is captured, that relates to patient care, may only be captured in REDCap temporarily and only under agreed circumstances, with the aim to migrate the functionality to the EMR (Cerner Millennium) environment as soon as it can be made to happen.

There have always been a large number of audits - clinical and non-clinical - going on in our health service at any given point in time. This is especially true since the Australian government instituted a new set of national standards for hospitals [25]. One of the effects of the combination of the instantiation of REDCap and the creation of the REASON platform is that setting up and collecting data for an audit is now more streamlined than ever. It also means however that there are now - completely predictable - governance requirements to he addressed around who gets to create audits on the platform and under what circumstances. Good independent governance, including assessing the need for a piece of work, exists for research but this is not necessarily so for clinical audit activities. One of the lessons of this work, which in part an inevitable consequence of the success of REDCap, is that there now needs to be greater consideration given to the governance around audits - most particularly clinical audits - as they are now easier than ever to create and run.

Another key lesson of the work, although not sometime that is that surprising, as that the success of the REDCap platform at AH has meant that it now needs some ongoing human resources to run it as a business service. Whilst we already have found and used existing skilled labour from within our current workforce to act in superuser- system expert roles, this has been to the detriment of other work streams at times. We ate currently discussing whether we can access a permanent dedicated staff member to act in this system expert role.

\subsection{Future Work}

As outlined in the section above on learnings, there is now work to be done at $\mathrm{AH}$ to consider the process by which audits - especially clinical audits - are approved; as well as work to be done to 
ensure that $\mathrm{AH} \mathrm{RCP}$ data collections are reused where possible, and that the same basic data elements are not re-collected in slightly different ways in subsequent audits.

Another of the next steps in this work program is to further expand the awareness of the capabilities of the RCP platform amongst health service staff. To this end we recently held 2 internal sessions about the platform, the successes it has supported, and the future opportunities it provides. Key business users of the system such as the Director in charge of nursing quality spoke at the session about their positive experiences with the platform and running projects (eg - clinical audit activities) on it.

One of the additional steps we have taken, with an eye to the future, is to embed a link to the RCP instance at $\mathrm{AH}$ within a broader intranet portal (Figure 5) that contains a number of other web based applications created or managed by the AKM department.

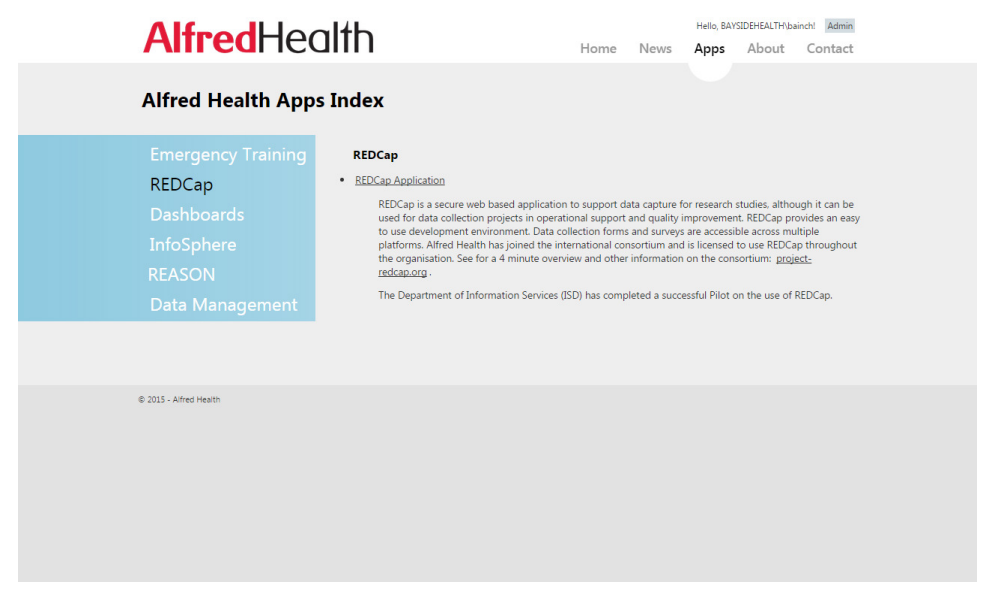

Figure 5. AKM Intranet Portal - Link to REDCap

Recently an organisation wide program around recognising excellence was supported by an inhouse built REDCap survey to allow the electronic nomination of teams or individuals to potentially receive recognition awards. This piece of RCP development can of course be reused in subsequent years, and can also serve as a robust permanent repository of the history of nominations and the reason for nomination, over time.

\section{Conclusions}

The acquisition, piloting and ongoing operationalisation of REDCap at $\mathrm{AH}$ has been an overwhelming success. Evidence of this is the vast number of projects being created and hosted on the platform, and the ongoing support we had from various business stakeholders for the platform. We recently held 2 seminars about the use of the platform at $\mathrm{AH}$ and the opportunities it provides for multiple stakeholders. These sessions were well supported by experienced and potential users of REDCap alike.

Next steps with the platform include more activities like the combined use of REASON and REDCap to support large research studies and audits - especially where existing data is already captured in REASON, thus saving researchers in particular, much time and money, as per the model used in the LICRA study. This in turn saves public monies that can better spent on more direct care activities 
Whilst in this case study we have described the benefits of REDCap in our context - that of a very large Australian healthcare organization - we believe that REDCap or platforms like it hold significant promise for smaller healthcare organizations that undertake research and audit activities. The vast number of members of the REDCap consortium internationally is also a testament to this.

\section{ACKNOWLEDGEMENTS}

The authors would like to thank the IDD teams that have assisted with the work on the AH RCP, and in particular the infrastructure team and the service delivery team. We would also like to thank Dr Ethan Gershon for his support of the acquisition of the RCP when it was first raised as a possibility for $\mathrm{AH}$, and Mr Emilio Pozo for his subsequent support. Finally we would like to thank the dozens of clinicians, business users and researchers who have seen the unique opportunity presented by REDCap and the REASON platform, and who have worked with us to make these initiatives a success. In particular we would like to acknowledge Suzanne Corcoran (Consumer Participation), Pam Ingram (Nursing Quality), Shirley Leong (Infection Prevention) and Dr David Mc Ilroy (Lead Anaesthesia clinician on the LICRA Study).

\section{REFERENCES}

[1] Alfred Health Website. http://www.alfred.org.au/ Accessed 11/3/2015

[2] D. Lazer, D. Kennedy, G. King, and A. Vespignani. 2014. The Parable of Google Flu: Traps in Big Data Analysis. Science 343 (6176) (March 14): 1203-1205.

[3] T.Murdoch and A.Detsky. The Inevitable Application of Big Data to Healthcare JAMA. 2013;309(13):1351-1352.

[4] C.Bain and C.Mac Manus. Advancing data management and usage in a major Australian health service: The REASON Discovery Platform TM. Proceedings of the International Conference on Data Science and Engineering 2014.

[5] N.Good, C.Bain, D.Hansen and S.Gibson. Health informatics visualisation engine - HIVE. Big Data Conference, Abstract Book. pp30-31. Big Data Conference, Melbourne April 2014.

[6] D.Martinez, L.Cavedon, Z.Alam, C.Bain and K. Verspoor. Text mining for lung cancer cases over large patient admission data. Big Data Conference, Abstract Book. Pp 24-25. Big Data Conference, Melbourne April 2014.

[7] C.Bain, T.Bucknall and J. Weir-Phyland. A clinical quality feedback loop supported by mobile pointof-care (POC) data collection. IMMoA Workshop 2013. Trento, Italy August 2013.CEUR Workshop Proceedings Vol 1075.pp44-51.

[8] C.Bain, T.Bucknall, J. Weir-Phyland, S.Metcalf. P.Ingram and L.Nie. Meeting National Safety and Quality Health Service Standards - The Role of the Point-of-Care Audit (POC) Application. IJEEEE 2013 Vol.3(6): pp 507-512.

[9] C. Bain, J. Weir-Phyland, S. Metcalf, P.Ingram and T.Bucknall. Driving clinical safety initiatives through innovative technological feedback systems in an Australian academic health service. - Oral Presentation. IARMM 2nd World Congress on Clinical Safety. Sep 12-12 2013. Heidelberg, Germany.

[10] Bain C, Mac Manus C and Seah J. Web Based Cohort Identification across Large Healthcare Data Sets - Opening the Treasure Chest. Proceedings of the International Conference on Computer Science, Data Mining and Mechanical Engineering - ICCDMME 2015, Bangkok, Thailand; April 2015.

[11] Seah J, Bain C and Mac Manus C. A Computational Platform for Radiology Image Retrieval and Analysis. Proceedings of the International Conference on Computer Science and Information Systems ICCSIS 2015. Pattaya, Thailand; April 2015.

[12] Bain C, Mac Manus C and Fitzgerald M. Efficiencies in healthcare data management - the case of clinical registries. Canadian International Journal of Science and Technology Volume 2, May 2015.

[13] Victorian State Trauma Outcomes Registry (VSTORM) Website. http://www.med.monash.edu.au/epidemiology/traumaepi/traumareg/. Accessed 11/3/2015 
Health Informatics - An International Journal (HIIJ) Vol.4, No.3/4, November 2015

[14] DEPM Website - Clinical Registries. http://www.med.monash.edu.au/epidemiology/unitscentres/registries/ .Accessed 10/3/2015

[15] I2B2 - https://www.i2b2.org/about/index.html. Accessed 24/12/2013

[16] STRIDE -https://clinicalinformatics.stanford.edu/research/stride.html Accessed 24/12/2013

[17] J.Frankovich, C.Longhurst and S.Sutherland. Evidence-Based Medicine in the EMR Era. N Engl J Med 2011; 365:1758-1759.

[18] REDCap Website - http://project-redcap.org/ Accessed 1/9/2015.

[19] Paul A. Harris, Robert Taylor, Robert Thielke, Jonathon Payne, Nathaniel Gonzalez, Jose G. Condee. Research electronic data capture (REDCap)_A metadata-driven methodology and workflow process for providing translational research informatics support. Journal of Biomedical Informatics 42 (2009) 377-381

[20] Active Directory - $\quad$ Microsoft Website. https://msdn.microsoft.com/enus/library/aa362244(v=vs.85).aspx . Accessed 15/9/2015.

[21] Murdoch Children's Research Institute Website - https://www.mcri.edu.au/ Accessed 1/9/2015.

[22] Microsoft Access 97 Support - Microsoft Website https://support.microsoft.com/enus/lifecycle?p1=1269\#gp/lifeobsoleteproducts Accessed 3/9/2015.

[23] United States of America Government Clinical Trials Website. Limiting IV Chloride to Reduce Acute Kidney Injury after Anaesthesia - LICRA. https://clinicaltrials.gov/ct2/show/NCT02020538 Accessed 16/9/2015.

[24] Cerner Millennium. https://www.cerner.com/default.aspx Accessed 1/9/2015.

[25] National Safety and Quality Health Service Standards Website. http://www.safetyandquality.gov.au/our-work/national-standards-and-accreditation/ Accessed $1 / 9 / 2015$. 